\title{
ON THE STRUCTURE OF CERTAIN LOCALLY COMPACT TOPOLOGICAL GROUPS
}

\author{
TA-SUN WU
}

Dedicated to Professor Karl H. Hofmann

\begin{abstract}
A locally compact topological group $G$ is called an $(H)$ group if $G$ has a maximal compact normal subgroup with Lie factor. In this note, we study the problem when a locally compact group is an $(\mathrm{H})$ group.
\end{abstract}

Let $G$ be a locally compact Hausdorff topological group. Let $G_{0}$ be the identity component of $G$. If $G / G_{0}$ is compact, then we say that $G$ is almost connected. The structure of almost connected locally compact groups is well understood and it is the consequence of the solution of the Hilbert fifth problem. Roughly speaking, the structure of almost connected locally compact groups can be described by the following statement: Every almost connected locally compact group has small normal subgroups such that the quotient groups are Lie groups and the group itself is the inverse limit of these Lie groups in a natural way. We cannot expect locally compact groups in general to have such properties, so we ask when a locally compact group has a compact normal subgroup such that the quotient group is a Lie group. The purpose of the present note is to provide some partial answers to this question.

Now, let $G$ be a locally compact group. We may view $G$ as an extension of $G_{0}$ by a totally disconnected locally compact topological group $G / G_{0}$. Since the natures of these two types of groups are very different, it is convenient to study them separately first and then put results together afterwards. Because our knowledge of connected locally compact groups is by far superior to what we know about totally disconnected locally compact groups, our main effort lies on totally disconnected locally compact groups. Actually, we do not lose any generality approaching our problem by this method. If $G / G_{0}$ has a compact normal subgroup such that the quotient group is a Lie group (i.e., a discrete group), then $G$ has an open normal subgroup $G_{1}$ such that $G_{1} / G_{0}$ is compact. Then $G_{1}$ has a maximal compact normal subgroup $K$ so that $G_{1} / K$ is a Lie group, since $G_{1}$ is almost connected. Since $K$ is characteristic in $G_{1}$, it is normal in $G$. Therefore $G / K$ is a Lie group. Notice that every totally disconnected locally compact group has small compact open subgroups. Frequently,

Received by the editors July 26, 1988 and, in revised form, May 3, 1989.

1980 Mathematics Subject Classification (1985 Revision). Primary 22A05. 
there are closed subgroups which are "almost" supplemented to the open compact subgroups. Roughly speaking this means that the given locally compact group $G$ has the form $H K$ such that $K$ is an open compact subgroup and $H \cap K$ has void interior.

Now, with this background in mind, it is natural to seek the conditions on the uniform subgroup $H$ so that there exist compact-open normal subgroups $K$ with $G=H K$. In the following, we shall more or less follow this direction. Our approach to this problem uses two basic notions in locally compact groups: compactly generated groups and bounded elements. Let $G$ be a topological group. Recall an element $x$ of $G$ is bounded if and only if $\left\{g x g^{-1}: g \in\right.$ $G\}$ is a relative compact subset of $G$. The subset $B(G)$ of all the bounded elements forms a characteristic subgroup of $G$. In recent years, we have learned a great deal about this subgroup [8,11-18]. Here, once again we have found its usefulness. In fact, implicitly, we have developed a type of relative version of boundedness.

Now, we sketch some results of the present article (Proposition 4):

Let $G$ be a totally disconnected solvable locally compact group. Let $H$ be a closed uniform subgroup of $G$. Let $H=D^{0}(H) \supset D^{(1)}(H) \supset \cdots \supset D^{(n)}(H)=$ (e) be the (topological) derived series of $H$. Assume that $D^{(i)}(H) / D^{(i+1)}(H)$ is compactly generated for $0 \leq i \leq n-1$. Then $G$ has a maximal compactopen normal subgroup. Furthermore, every closed subgroup of $G$ is compactly generated.

With the boundedness condition, we can say much more (Proposition 10):

Let $G$ be a locally compact totally disconnected compactly generated group. Let $H$ be a closed uniform solvable subgroup of $G$. Let $H=D^{0}(H) \supset$ $D^{(1)}(H) \supset \cdots \supset D^{(n)}(H)=(e)$ be the (topological) derived series of $H$. Assume that $D^{(i)}(H) / D^{(i+1)}(H) \subset B\left(H / D^{(i+1)}(H)\right)$ for $0 \leq i \leq n-1$. Then $G$ has a maximal compact-open normal subgroup $E$. Every closed subgroup of $G$ is compactly generated. Furthermore $G / E$ is a discrete nilpotent by finite group.

For locally compact groups in general, we have the following condition (Proposition 11):

Let $G$ be a locally compact group. If $G / G_{0}$ has a maximal compact-open normal subgroup, then $G$ has a maximal compact-normal subgroup $K$ such that $G / K$ is a Lie group.

There are examples which show the converse of Proposition 11 is not true; however, we have some partial converses (Propositions 14, 15, 16). Here, we exploit the relations between $G$ and $G_{0}$. Specifically, we try to use as much as possible the linear representation of $G$ on the Lie algebra $\mathscr{L}\left(G_{0}\right)$ of $G_{0}$. We give several examples which indicate these results are likely to be the best possible along this line, especially Example 21 which shows a certain complexity between the relation of $G$ and $G_{0}$ when we view $G$ as a subgroup of $\mathrm{GL}\left(\mathscr{L}\left(G_{0}\right)\right)$. 
In view of the importance of the boundedness conditions, an attempt is made to see what the key features are which make it useful. We introduce the definition of weak boundedness. An element $x$ in $G$ is weakly bounded if and only if the smallest closed normal subgroup $N(x)$ of $G$ which contains $x$ has the following properties: (1) $N(x)$ has a maximal compact normal subgroup $K(x)$ such that $N(x) / K(x)$ is a Lie group, and (2) every closed subgroup of $N(x)$ is compactly generated. Every bounded element is weakly bounded. Let $B^{*}(G)$ denote the subset of all weakly bounded elements of $G$. Then we have the following results (Proposition 26 and Remark 27):

Let $H$ be a totally disconnected locally compact group. Assume that $H$ has a finite sequence of closed normal subgroups $H=H_{1} \supset H_{2} \supset \cdots \supset H_{n} \supset(e)$ such that (1) $H_{i} / H_{i+1}$ is compactly generated, and (2) $H_{i} / H_{i+1} \subset B^{*}\left(H / H_{i+1}\right)$ for $1 \leq i \leq n$. Then $H$ is an $(\mathrm{H})$ group and every closed subgroup is compactly generated. If $H$ is a normal uniform subgroup of a locally compact group $G$, then $G$ is an $(\mathrm{H})$ group and every closed subgroup of $G$ is compactly generated (cf. [9]). (A locally compact group $G$ is an (H) group if it has a maximal compact normal subgroup $K$ such that $G / K$ is a Lie group.)

The following result characterizes the existence of an open invariant neighborhood of the identity (Corollary 32 ):

Let $G$ be a totally disconnected locally compact group. Then $G$ has a compact open normal subgroup if and only if $B^{*}(G)$ is an open subgroup (cf. [16]).

Now, one may ask what is the significance of a subgroup of $B(G)$ being locally compact. The following propositions give some answers to this question. Specifically, when $D$ is a compact group of bounded elements, we show that the smallest closed normal subgroup $N(D)$ of $G$ which contains $D$ is compact (Propositions 35, 37, and 38):

Let $G$ be a locally compact totally disconnected group. Let $D$ be a compact subset of $B(G) \cap P(G)$. Then the smallest closed normal subgroup $N(D)$ of $G$ which contains $D$ is compact if and only if $N(D) \subset B(G)$. If $D$ is a compact subgroup of $B(G)$, then $N(D) \subset B(G)$ and it is a compact group. Let $E$ be a compact subset of $B(G)$. Then the smallest closed normal subgroup $N(E)$ of $G$ which contains $E$ is compactly generated if $E \subset B(G)$ (Corollary 39):

Let $G$ be a totally disconnected locally compact group. Then $B(G)$ is locally compact if and only if $P(G) \cap B(G)$ is locally compact.

In our discussions, we have singled out a significant class of locally compact groups, namely, those which have a maximal compact normal subgroup such that the quotient group is a Lie group. We shall call such a locally compact group an $(\mathrm{H})$ group. (H stands for Karl H. Hofmann. After reading through an original version of the manuscript [4], Hofmann points out to us this class of locally compact groups. This beautiful notion already proves its usefulness in the present article.)

1. Standing notation. All the topological groups considered in this note will have Hausdorff topology. Let $G$ be a locally compact group. The identity 
component of $G$ will be denoted by $G_{0}$. The identity of $G$ will be denoted by $e$. Let $K$ be a subset of $G$. The subgroup generated by $K$ will be denoted by $\langle K\rangle$. Then $\langle K\rangle=\bigcup_{n=1}\left(K \cup K^{-1}\right)^{n}$. If $K$ is compact, and $G=\langle K\rangle$, then we say that $G$ is compactly generated. However, if $G=\langle K\rangle^{-}$, i.e., $\langle K\rangle$ is a dense subgroup of $G$, then for any subset $V$ of $G$ with nonvoid interior, we have that $G=\langle K\rangle V$. When $G$ is locally compact, we may choose $V$ to be any compact neighborhood (at any point). Then $G=\langle K \cup V\rangle$, and $G$ is compactly generated. Therefore, for locally compact groups, if there exists a compact subset which generates a dense subgroup, then this group is compactly generated. Let $B(G)$ denote the subgroup of all bounded elements of $G$, and let $P(G)$ be the subset of all compact elements of $G$. An element $x$ of $G$ is compact, i.e., $x \in P(G)$, if the smallest closed subgroup which contains $x$ is compact.

Finally, a topological group $G$ is an $(\mathrm{H})$ group if and only if it has a maximal compact normal subgroup $E$ such that $G / E$ is a Lie group [4]. When $G$ is totally disconnected, $G$ is an $(\mathrm{H})$ group if and only if it has a maximal compact normal open subgroup (so the quotient group is a discrete group).

2. Lemma. Let $G$ be a totally disconnected locally compact solvable group. Let $G=D^{(1)}(G) \supset D^{(2)}(G) \cdots \supset D^{(n)}(G)=(e)$ be the topological derived series of $G$. Assume that $D^{(i)}(G) / D^{(i+1)}(G)$ is compactly generated. Then $G$ is an $(\mathrm{H})$ group and every closed subgroup of $G$ is compactly generated. (Notation: $\mathscr{D}^{(1)}(G)=$ $\left.\langle[G, G]\rangle, \mathscr{D}^{(i)}(G)=\left\langle\left[\mathscr{D}^{(i-1)}(G), \mathscr{D}^{(i-1)}(G)\right]\right\rangle, D^{(i)}(G)=\mathscr{D}^{(i)}(G)^{-}.\right)$

Proof. We prove this lemma by induction on the length of the derived series. When the length is one, $G$ is an abelian compactly generated locally compact group. The assertions of the lemma follow from the structure theorem of compactly generated locally compact abelian groups. Now, we assume the lemma is true when the length of the derived series is less than or equal to $n-1$. Let $F=G / D^{(n-1)}(G)$. Then $F$ has a maximal compact normal subgroup $Q$ such that $F / Q$ is discrete. Let $H$ be the inverse image of $Q$ in $G$. Then $D^{(n-1)}(G)$ is uniform in $H$. Now, $D^{(n-1)}(G)$ is a compactly generated locally compact abelian group by assumption, so it has a maximal compact subgroup $K$. Since $D^{(n-1)}(G)$ is normal in $G$ and $K$ is a characteristic subgroup of $D^{(n-1)}(G)$, it follows that $K$ is normal in $G$. Let $G^{\prime}=G / K, H^{\prime}=H / K$, and $L^{\prime}=D^{(n-1)}(G) / K$. Then $L^{\prime}$ is a finitely generated discrete abelian group. Therefore the automorphism group $\mathscr{A}\left(L^{\prime}\right)$ is a discrete group. Let $\rho$ be the representation of $G^{\prime}$ on $L^{\prime}$ by conjugations (restriction of inner automorphisms on $\left.L^{\prime}\right)$. Then $\rho\left(H^{\prime}\right)$ is compact, so it is finite. Let $M^{\prime}$ be the kernel of $\rho$ when $\rho$ is restricted to $H^{\prime}$, i.e., $M^{\prime}=($ kernel of $\rho) \cap H^{\prime}$. Then $H^{\prime} / M^{\prime}$ is finite and $L^{\prime} \subset M^{\prime}$. Therefore $M^{\prime}$ is a compactly generated $(Z)$ group (i.e., central group). By the structure theorem of central groups [7], $M^{\prime}$ has a maximal compact normal group $P^{\prime}$. Now, let $\pi$ be the canonical homomorphism $G \rightarrow G / K$, and let $\pi^{-1}\left(P^{\prime}\right)=P$, where $P$ is a compact subgroup. It is not 
difficult to see that $P$ is a maximal compact open normal subgroup of $G$. Now, we show that every closed subgroup $N$ of $G$ is compactly generated. From the isomorphism $N / N \cap P \cong N P / P \subset G / P$, we may assume that $G$ is discrete and we show that $N$ is finitely generated. Again, we prove this by induction on the length of the derived series. So, (by the inductive hypothesis) assume $N D^{(n-1)}(G) / D^{(n-1)}(G)$ is finitely generated. Since $N / N \cap D^{(n-1)}(G)$ is isomorphic to $N D^{(n-1)}(G) / D^{(n-1)}(G)$, it follows that $N / N \cap D^{(n-1)}(G)$ is finitely generated. Since $N \cap D^{(n-1)}(G)$ is finitely generated, a fortiori, $N$ is finitely generated. Therefore, the proof is complete.

3. Lemma. Let $G$ be a locally compact topological group. Let $H$ be a totally disconnected solvable subgroup of $G$ such that (1) $H$ is uniform in $G$, and (2) $D^{(i)}(H) / D^{(i+1)}(H)$ is compactly generated for $1 \leq i \leq n-1$, where $n$ is the length of the derived series of $H$. Then there is a compact normal subgroup $K$ of $G$ such that $\pi(H)$ is discrete in $\pi(G)$, where $\pi$ is the canonical homomorphism $G \rightarrow G / K$. Furthermore $D^{(i)}(\pi(H)) / D^{(i+1)}(\pi(H))$ is finitely generated, $1 \leq i \leq$ $n-1$.

Proof. By Lemma 2, $H$ has a maximal compact open normal subgroup $M$. Since $H$ is uniform in $G$, there is a compact subset $Q$ of $G$ such that $G=H Q$. Since $M$ is compact and normal in $H, \bigcup\left\{x M x^{-1}: x \in Q\right\}=$ $\bigcup\left\{x M x^{-1}: x \in H Q\right\}$ is compact, therefore $M \subset B(G)$. Let $K$ be the closed normal subgroup generated by $M$. Then $K$ is a compact subgroup of $G$ [16]. Since $K \cap H$ is normal in $H, K \cap H=M$. Therefore $H K / K \cong H / H \cap K=$ $H / M$ and $D^{(i)}(\pi(H)) / D^{(i+1)}(\pi(H))$ is a finitely generated abelian group for $1 \leq i \leq n-1$.

4. Proposition. Let $G$ be a totally disconnected solvable locally compact group. Let $H$ be a closed uniform subgroup of $G$ with the property: $D^{(i)}(H) / D^{(i+1)}(H)$ is compactly generated for $1 \leq i \leq m$, where $m+1$ is the length of the derived series of $H$. Then $G$ is an $(\overline{\mathrm{H}})$ group and every closed subgroup of $G$ is compactly generated.

Proof. Let $m+1$ be the length of the derived series of $G$. Then $D^{(m)}(G)$ is an abelian group. We show that $D^{(m)}(G)$ is compactly generated. Let $F=\left(H D^{(m)}(G)\right)^{-}$. Then $H$ is a uniform subgroup of $F$. By Lemma 3, there is a compact normal subgroup $L$ of $G$ such that, in the quotient group $G / L$, the image of $H$ is a finitely generated discrete subgroup. For simplicity of notation, we may assume that $H$ is a discrete subgroup of $F$. Now $H D^{(m)}(G)$ is a dense subgroup of $F$, so $K H D^{(m)}(G)=F$ for any compact open subgroup $K$ of $F$. We have the following isomorphism: $K D^{(m)}(G) / H \cap K D^{(m)}(G) \rightarrow$ $H K D^{(m)}(G) / H$. Let $E=H \cap K D^{(m)}(G)$. Then $E$ is a discrete finitely generated uniform subgroup of $K D^{(m)}(G)$. Hence $K D^{(m)}(G)$ is compactly generated. This implies that $D^{(m)}(G)$ is compactly generated, since it is a uniform subgroup of $K D^{(m)}(G)$. 
Now, we may factor out $D^{(m)}(G)$ from $G$ and proceed to show that $D^{(m-1)}(G)$ is compactly generated. Certainly, in this case, we need to consider $\left(H D^{(m)}(G)\right)^{-} / D^{(m)}(G)$ instead of $H$ itself. So, we need to verify that

$$
\left(H D^{(m)}(G)\right)^{-} / D^{(m)}(G)
$$

enjoys the same properties as $H$. But this is easy to check since the image of $H$ in $\left(H D^{(m)}(G)\right)^{-} / D^{(m)}(G)$ is a dense subgroup. So, $D^{(i)}(G) / D^{(i+1)}(G)$ is compactly generated for $1 \leq i \leq m$. By Lemma $2, G$ is an $(\mathrm{H})$ group and every closed subgroup of $G$ is compactly generated.

5. Proposition. Let $G$ be a totally disconnected locally compact group. Suppose there exist a finite sequence of closed compactly generated normal subgroups $G$, $G=G_{1} \supset \cdots \supset G_{n}$, such that $G_{i} / G_{i+1} \subset B\left(G / G_{i+1}\right)$ for $1 \leq i \leq n-1$. Then $G$ is an $(\mathrm{H})$ group. Let $K$ be the maximal compact normal subgroup of $G$. Then $G / K$ is a discrete nilpotent-by-finite group.

Proof. First, we show that $G$ is an $(\mathrm{H})$ group. We prove this by induction on the length of the finite sequence. When the length is one, $G=G_{1}=B(G)$ is a compactly generated $\overline{\mathrm{FC}}$ group. It is known that $G$ has a maximal compact normal subgroup $K$ and $G / K$ is a torsion free finitely generated abelian group. Hence $G$ is an (H) group. Now, let $F=G / G_{n}$ and let $\pi$ be the canonical homomorphism $G \rightarrow G / G_{n}$. By the induction hypothesis, $F$ has a maximal normal compact open subgroup $M^{\prime}$. Let $M=\pi^{-1}\left(M^{\prime}\right)$. We have $G_{n} \subset M \subset$ $G$ and $M / G_{n}$ is compact. Now, we can use the same argument as we did in the proof of Lemma 2 to conclude that $G$ has a maximal compact open normal subgroup $K$ so that $G / K$ is a discrete group. Now, we shall prove that $G / K$ is a nilpotent-by-finite group. For the sake of simplicity of notation, we may assume that $K$ is trivial and $G$ is a discrete group. We shall prove this by induction. Since $G_{n}$ is a discrete finitely generated torsion free abelian group, $G_{n}$ is isomorphic to $Z^{m}$ for some $m$, and its group of automorphisms is isomorphic to $\operatorname{GL}(m, Z)$. Let $\rho$ be the representation of $G$ on $G_{n}$ by the inner automorphisms. Since $G_{n} \subset B(G), \rho(G)$ is relative compact, and since Aut $G_{n}$ is discrete, $\rho(G)$ is finite. Therefore the kernel $G^{\prime}$ of $\rho$ is a subgroup of finite index in $G$. Since $G_{n}$ is abelian, $G_{n} \subset$ kernel of $\rho=G^{\prime}$. Therefore $G_{n}$ is central in $G^{\prime}$. Now, by the induction hypothesis, $G / G_{n}$ has a subgroup which is nilpotent-by-finite. Let $G^{\prime \prime}$ be the inverse image of that nilpotent subgroup. Then $G_{n} \subset G^{\prime \prime}$ and $G^{\prime \prime}$ has finite index in $G$. It is clear that $G_{n} \subset G^{\prime} \cap G^{\prime \prime}, G_{n}$ is central in $G^{\prime} \cap G^{\prime \prime}$, and $G^{\prime} \cap G^{\prime \prime}$ has finite index in $G$. So $G^{\prime} \cap G^{\prime \prime}$ is nilpotent. This completes the proof.

So far, we have assumed the groups involved in our discussions are built up by compactly generated subgroups. The proofs of most previous statements are based upon the induction from the bottom of the derived series. This means we already have the condition "compactly generated" at our disposal. Now, if we only assume that the group $G$ is compactly generated, in view of the existence of 
compactly generated groups with noncompactly generated subgroups, the matter becomes much more delicate than before (cf. Example 18).

6. Lemma. Let $G$ be a totally disconnected solvable compactly generated group. Suppose $D^{(1)}(G)$ is abelian and a subgroup of $B(G)$. Then $D^{(1)}(G)$ is compactly generated. Furthermore, $G$ is an $(\mathrm{H})$ group. Let $E$ be the maximal compact normal subgroup of $G$. Then $G / E$ is nilpotent-by-finite.

Proof. Let $X$ be a compact-open subgroup of $D^{(1)}(G)$. Let $N(X)$ be the smallest closed normal subgroup of $G$ which contains $X$. By Proposition 35 or 38, $N(X)$ is a compact-normal subgroup of $G$ which is open in $D^{(1)}(G)$. Let $G^{\prime}=G / N(X)$. Then $D^{(1)}\left(G^{\prime}\right)$ is a discrete abelian subgroup of $G^{\prime}$. If $D^{(1)}\left(G^{\prime}\right)$ is compactly generated, then $D^{(1)}(G)$ is compactly generated. In order to show that $D^{(1)}\left(G^{\prime}\right)$ is compactly generated we only need to prove that $\mathscr{D}^{(1)}\left(G^{\prime}\right)$ is compactly generated since the latter is a dense subgroup of $D^{(1)}(G)$. Now, $G^{\prime}$ is compactly generated, so $G^{\prime}=\bigcup_{n=1}^{\infty} K^{\prime n}$ for some compact subset $K^{\prime}$ of $G^{\prime}$. Since we have the equality $[x y, z]=[x, z]^{y}[y, z]$ for $x, y, z \in K^{\prime}, \mathscr{D}^{(1)}\left(G^{\prime}\right)$ is generated by $\left[K^{\prime}, K^{\prime}\right]^{G^{\prime}}$. Because $\left[K^{\prime}, K^{\prime}\right] \subset D^{(1)}\left(G^{\prime}\right) \subset B\left(G^{\prime}\right),\left[K^{\prime}, K^{\prime}\right]^{G^{\prime}}$ is finite, since $D^{(1)}\left(G^{\prime}\right)$ is discrete and $[K, K]$ is finite. Hence $D^{(1)}\left(G^{\prime}\right)$ is finitely generated. Therefore $D^{(1)}(G)$ is compactly generated. The rest of the assertions follow from Proposition 5.

7. Lemma. Let $G$ be a compactly generated totally disconnected locally compact solvable group. Suppose $D^{(2)}(G)$ is an abelian group and $D^{(2)} \subset B(G)$. We also assume that $D^{(1)}(G) / D^{(2)}(G) \subset B\left(G / D^{(2)}(G)\right)$. Then $D^{(1)}(G)$ is compactly generated.

Proof. Let $Q$ be a compact open subgroup of $D^{(2)}(G)$. Since $D^{(2)}(G) \subset B(G)$ by Proposition 35, $Q$ generates a compact normal subgroup $Q^{\prime}$ of $G$. If we factor out $Q^{\prime}$ from $G$, then $D^{(2)}(G) / Q^{\prime}$ is a discrete abelian group. Since we wish to show that $D^{(1)}(G)$ is compactly generated, factoring out a compact normal subgroup from $G$ clearly will not change the status of $D^{(1)}(G)$, so we shall assume that $D^{(2)}(G)$ is a discrete abelian group without loss of any generality. Now, by Lemma $6, G / D^{(2)}(G)$ has a maximal compact normal subgroup $E$ such that $\left(G / D^{(2)}(G)\right) / E$ is nilpotent-by-finite. It is clear that we may assume $\left(G / D^{(2)}(G)\right) / E$ is nilpotent without changing the status of $D^{(1)}(G)$.

Let $F$ be the inverse image of $E$ in $G$. Since $F / D^{(2)}(G) \cong E$ is compact, $F=L D^{(2)}(G)$ with $L$ a compact subset of $G$. So, we have the following situation: (1) $D^{(2)}(G) \subset F=L D^{(2)}(G) \subset G$, (2) $[G,[G, G]] \subset F,(3) G$ is compactly generated, and (4) $D^{(2)}(G) \subset B(G)$. Now, let $K$ be a compact subset of $G$ which generates $G$, i.e., $G=\bigcup_{n=1}^{\infty} K^{n}, K=K^{-1} \supset e$. Since $[K,[K, K]]$ is a compact subset of $F, F=L D^{(2)}(G)$, there exists a compact subset $S_{1}$ of $D^{(2)}(G)$ such that $[K,[K, K]] \subset L S_{1}$. Since $[K, L]$ is a compact subset of $F$, there exists a compact subset $S_{2}$ of $L$ such that $[K, L] \subset L S_{2}$. 
Let $S=S_{1} \cup S_{2}$ (in fact, $D^{(2)}(G)$ is discrete, $S$ is a finite set). Then we have (i) $[K, L] \subset L S$, and (ii) $[K,[K, K]] \subset L S$. Since $S \subset D^{(2)}(G) \subset B(G)$, $S^{G}$ is finite. Now $\mathscr{D}^{(1)}(G)=\langle[G, G]\rangle=\left\langle\left[\bigcup K^{n}, \bigcup K^{n}\right]\right\rangle$ is generated by the elements of the form $\left[k_{1} \cdots k_{m}, k_{1}^{\prime} \cdots k_{n}^{\prime}\right], k_{i}, k_{j}^{\prime} \in K$. Using the equality $\left[k_{1} k_{2}, k\right]=\left[k_{2},\left[k_{1} k\right]\right]\left[k_{1}, k\right]\left[k_{2}, k\right]$ and relations (i) and (ii), we have the subset $[G, G]$ is contained in the subgroup generated by $X=[K, K] \cup L \cup S^{G}$. Since $X$ generates $F D^{(1)}(G)=L D^{(1)}(G), L D^{(1)}(G)$ is compactly generated. Since $D^{(1)}(G)$ is uniform in $L D^{(1)}(G), D^{(1)}(G)$ is compactly generated and the proof is complete.

Observe the group $G$ in Lemma 7 is an $(\mathrm{H})$ group. Let $E$ be the maximal compact normal subgroup of $G$. Then $G / E$ is a discrete nilpotent-by-finite group. Actually, this is true in a more general situation. We state it as the next lemma.

8. Lemma. Let $G$ be a compactly generated totally disconnected locally compact solvable group. Let $G=D^{(1)}(G) \supset \cdots \supset D^{(n)}(G)=(e)$ be the topological derived series of $G$. Assume $D^{(i)}(G) / D^{(i+1)}(G) \subset B\left(G / D^{(i+1)}(G)\right)$ for $1 \leq i \leq n-1$. Then $G$ is an $(\mathrm{H})$ group. Let $E$ be a maximal compact normal subgroup of $G$. Then $G / E$ is a discrete nilpotent-by-finite group.

Proof. With the help of Proposition 5 the proof can be carried out in the same manner as the proof of Lemma 7.

9. Lemma. Let $G$ be a totally disconnected locally compact group. Let $H$ be a compactly generated nilpotent uniform subgroup of $G$. Then $G$ is an $(\mathrm{H})$ group.

Let $E$ be the maximal compact normal subgroup of $G$. Then $G / E$ is a discrete nilpotent-by-finite group.

Proof. This in one of the main results in [4]. We shall prove this lemma by induction on the length of the upper central series of $H$.

Let $Z$ be the center of $H$. Then $Z \subset B(H) \subset B(G)$, since $H$ is uniform in $G$. Let $Q$ be the smallest closed normal subgroup of $G$ which contains $Z$. It is well known that $Q$ is an extension of a compact normal subgroup $M$ by a finitely generated torsion-free abelian group [16]. Let $G_{1}=G / Q$ and $H_{1}=\overline{H Q} / Q$. Then the length of the upper central series of $H_{1}$ is less than the length of the central series of $H$, so the induction hypothesis is applicable. Therefore $G_{1}$ has a maximal compact open normal subgroup $P_{1}$. Let $P$ be the complete inverse image of $P_{1}$ in $G$. Then we have the following conditions: (1) $P$ is open and normal in $G$, (2) $M \subset Q \subset P$ and $P / Q$ is compact, (3) $M$ is a maximal compact normal subgroup of $Q$, and (4) $Q / M \cong Z^{l}$ for some $l$, i.e., it is a torsion-free abelian group of rank $l$ for some integer $l$. Since $Q$ is normal in $G, M$ is characteristic in $Q$, so $M$ is a normal subgroup of $G$. Let $\pi$ be the quotient map of $P$ onto $P^{\prime}=P / M$ and let $Q^{\prime}=\pi(Q)=Q / M \cong Z^{l}$. Then $Q^{\prime}$ is a uniform subgroup of $P^{\prime}$. Using the same argument as that in the 
proof of Lemma 3, we have a maximal open normal subgroup $E^{\prime}$ of $P^{\prime}$. Let $E=\pi^{-1}\left(E^{\prime}\right)$. Then $E$ is a maximal compact open normal subgroup of $G$. Since $G / E$ is discrete and $H E / E$ is a nilpotent subgroup of $G / E$ with finite index, $G / E$ is a discrete nilpotent-by-finite group.

10. Proposition. Let $G$ be a compactly generated totally disconnected locally compact group. Let $H$ be a uniform solvable subgroup of $G$. Suppose

$$
D^{(i)}(H) / D^{(i+1)}(H) \subset B\left(H / D^{(i+1)}(H)\right)
$$

for $1 \leq i \leq n-1$, where $n$ is the length of the derived series of $H$. Then $G$ is an $(\mathrm{H})$ group.

Let $E$ be a maximal compact normal subgroup of $G$. Then $G / E$ is a discrete nilpotent-by-finite group.

Proof. Since $H$ is uniform in $G, H$ is compactly generated. By Lemma 8 , $H$ is an $(\mathrm{H})$ group and it has a maximal compact normal subgroup $M$ so that $H / M$ is a nilpotent-by-finite group. Since $M \subset B(H) \subset B(G)$, it follows that $M$ is contained in a compact normal subgroup $Q$ of $G$ [16]. Let $G_{1}=G / Q$ and $H_{1}=Q H / Q$. Since $Q \cap H=M, H_{1}$ is a nilpotent-by-finite group. Apparently, Lemma $9^{\prime}$ can be applied here. Let $E_{1}$ be the maximal compact subgroup of $G_{1}$ and let $E$ be the complete inverse image of $E_{1}$ in $E$. Then it is not difficult to see that $E$ is an open maximal compact normal subgroup of $G$ and so the proof is complete.

11. Proposition. Let $G$ be a locally compact group. If $G / G_{0}$ is an $(\mathrm{H})$ group, then $G$ is an $(\mathrm{H})$ group.

Proof. Let $\pi$ be the canonical homomorphism from $G$ onto $G^{\prime}=G / G_{0}$. Let $M^{\prime}$ be the maximal compact normal subgroup of $G^{\prime}$, and let $M=\pi^{-1}\left(M^{\prime}\right)$. Then $G_{0}$ is uniform in $M$. Since $M$ is almost connected, it has a maximal compact normal subgroup $Q$. Since $M$ is normal in $G$ and $Q$ is characteristic in $M, Q$ is normal in $G$. We claim that $Q$ is a maximal compact normal subgroup of $G$. Let $K$ be any compact normal subgroup of $G$. Since $\pi(K)$ is normal in $\pi(G)=G^{\prime}$, by the maximality of $M^{\prime}, \pi(K) \subset M^{\prime}$. Therefore $K \subset M$. Hence $K \subset Q$, and it follows that $Q$ is a maximal compact normal subgroup of $G$. Because $M$ is almost connected, $M / Q$ is a Lie group. Since $M$ is an open subgroup of $G$, a fortiori, $G / Q$ is a Lie group. So $G$ is an $(\mathrm{H})$ group.

12. Remark. The converse of Proposition 11 is not true, i.e., a locally compact group $G$ could be an $(\mathrm{H})$ group and $G / G_{0}$ not an $(\mathrm{H})$ group. The following is such an example. Let $\mathbf{C}^{*}$ be the multiplicative group of all nonzero complex numbers. Let $S$ be the subgroup of $\mathbf{C}^{*}$ consisting of $2^{n}$ th roots of unity, $n \geq 0$, with discrete topology. Let $G$ be the semidirect product of $S \cdot \mathrm{C}$, where $\mathbf{C}$ is the complex numbers. Then $G$ has no nontrivial compact normal subgroup, thus $G$ is an (H) group. But $G / G_{0}$ is an infinite torsion group and it is not an $(\mathrm{H})$ group. We note the group $G$ is a solvable group and is not 
compactly generated. However, we do have the following conditions. Let $G$ be a locally compact group. If $G$ has a compact normal subgroup $K$ such that $G / K$ is a Lie group, then $G^{\prime}=G / G_{0}$ has a compact normal subgroup $K^{\prime}$ such that $G^{\prime} / K^{\prime}$ is a discrete group. We simply take $K^{\prime}$ to be the canonical image of $K$ in $G^{\prime}$.

Now, let $\phi$ be the adjoint representation of $G$ on the Lie algebra $\mathscr{L}\left(G_{0}\right)$ of $G_{0}, \phi: G \rightarrow \operatorname{GL}\left(\mathscr{L}\left(G_{0}\right)\right)$. In general, $\phi(G)$ is not closed. When it is closed, then we shall see that $G / G_{0}$ is an $(\mathrm{H})$ group if $G$ is an $(\mathrm{H})$ group. On the other hand, even if $G$ is compactly generated, it is not enough that $G / G_{0}$ be an (H) group (Example 21). In the next few sections, our main theme is to find the connections between $G$ and its natural representation on $\mathscr{L}\left(G_{0}\right)$. To simplify the matter a little first, we give a simple lemma.

13. Lemma. Let $G$ be a locally compact group and let $Q$ be a compact normal subgroup of $G$. Then $G$ is an $(\mathrm{H})$ group if and only if $G / Q$ is an $(\mathrm{H})$ group. In particular, when $G$ is an $(\mathrm{H})$ group $G / G_{0}$ is an $(\mathrm{H})$ group if and only if $G^{\prime} / G_{0}^{\prime}$ is an $(\mathrm{H})$ group, where $G^{\prime}=G / K$ and $K$ is the maximal compact normal subgroup of $G$.

Proof. The first statement is obvious. For the second statement, simply observe that $G / G_{0} K$ is isomorphic to $G^{\prime} / G_{0}^{\prime}$ and the kernel of the map $G / G_{0}$ to $G / G_{0} K$ is compact.

14. Proposition. Let $G$ be a $\sigma$-compact locally compact (H) group. Assume the adjoint representation of $G$ on $G_{0}$ has a closed image. Then $G / G_{0}$ is an (H) group.

Proof. Let $\pi$ be the canonical homomorphism from $G$ onto $G / G_{0}$. Let $G_{1}=$ $\pi^{-1}\left(B\left(G / G_{0}\right) \cap P\left(G / G_{0}\right)\right)$. Then $G_{1}$ is generated by all the normal subgroups $G_{\lambda}$ of $G$ such that $G_{\lambda} / G_{0}$ is a compact normal subgroup of $G / G_{0}$. Thus, $G / G_{0}$ is an (H) group if and only if $G_{1} / G_{0}$ is compact. We know that $G$ is an $(\mathrm{H})$ group by assumption. By Lemma 13, we may assume the maximal compact normal subgroup of $G$ is trivial, so $G$ is a Lie group. Let $\phi$ be the adjoint representation of $G$ on the Lie algebra of $\mathscr{L}\left(G_{0}\right)$ of $G_{0}$. So $\phi: G \rightarrow$ $\mathrm{GL}\left(\mathscr{L}\left(G_{0}\right)\right)$ and $\phi(G)$ is closed by assumption. Since $G_{1}=\bigcup_{\lambda \in \Lambda} G_{\lambda}$, where $G_{\lambda} / G_{0}$ is a finite normal subgroup of $G / G_{0}, G_{\lambda}=G_{0} M_{\lambda}$ with $M_{\lambda}$ a maximal compact subgroup of $G_{\lambda}$. Since the maximal compact normal subgroup of $G$ is trivial, the centralizer of $G_{0}$ meets $M_{\lambda}$ trivially for every $\lambda \in \Lambda$, i.e., $Z_{G}\left(G_{0}\right) \cap M_{\lambda}=\{e\}$. To see the last statement, let $Z_{\lambda}=Z_{G}\left(G_{0}\right) \cap G_{\lambda}$. Since $G_{0}$ is open in $G_{1}, Z_{\lambda} / Z_{\lambda} \cap G_{0} \cong Z_{\lambda} G_{0} / G_{0}$, it follows that $Z_{\lambda} / Z_{\lambda} \cap G_{0}$ is finite and is a $(Z)$ group. Because the center of the analytic group is compactly generated, $Z_{\lambda}$ is a compactly generated $(Z)$ group. By the structure of compactly generated $(Z)$ groups, $Z_{\lambda}$ has a maximal compact normal subgroup $K_{\lambda}$ which certainly is characteristic in $G_{\lambda}$, so it is normal in $G$. Hence $K_{\lambda}$ is trivial. Therefore $Z_{\lambda} \cap M_{\lambda}=(e)$ for each $\lambda \in \Lambda$. So $\phi \mid M_{\lambda}$ is injective. By assumption, $\phi(G)$ is closed, so $\phi\left(G_{1}\right)=\bigcup_{\lambda \in \Lambda} \phi\left(G_{0}\right) \phi\left(M_{\lambda}\right) \subset \mathrm{GL}\left(\mathscr{L}\left(G_{0}\right)\right)$. Since $\operatorname{GL}\left(\mathscr{L}\left(G_{0}\right)\right)$ has 
a maximal compact subgroup, by conjugacy, we may assume that $\phi\left(M_{\lambda}\right)$ is contained in this maximal compact subgroup. Now, we have $\phi\left(G_{1}\right) / \phi\left(G_{0}\right)$ is compact. Therefore $G_{1} / G_{0}$ is compact and the proof is complete.

For the next proposition we need a definition. Let $G$ be an analytic group. Let $N$ be the maximal connected normal solvable subgroup of $\langle[G, G]\rangle^{-}$. The subgroup $N$ is called the representation radical of $G$.

In view of Lemma 13, when we consider the representation of $G$ on $G_{0}$ defined by conjugations, we may assume that $G_{0}$ is an analytic group without loss of generality. We shall do so in the next two propositions.

15. Proposition. Let $G$ be a compactly generated locally compact $(\mathrm{H})$ group with trivial compact normal subgroup so that $G$ is a Lie group. Let $\phi$ be the linear representation of $G$ on the Lie algebra $\mathscr{L}\left(G_{0}\right)$ of $G_{0}$, let $\phi(G)^{*}$ be the Zariski closure of $\phi(G)$, and let $N$ be the representation radical of $G_{0}$. If the representation radical of $\phi(G)^{*}$ (as an analytic group) is the same as $\phi(N)$, the $G / G_{0}$ is an $(\mathrm{H})$ group.

Proof. We follow the same notation as given in Proposition 14. As we already observed in Proposition 14, $\phi \mid M_{\lambda}$ is faithful. For each $\lambda, G_{\lambda}=G_{0} M_{\lambda}=G_{0} D_{\lambda}$, where $D_{\lambda}$ is a finite subgroup of $G_{\lambda}$. Since $\phi(G)^{*}$ has finite index in $\phi(G)^{*}$, we may assume that $\phi(G)$ is a subgroup of $\phi(G)^{*}$. Let $S$ be a Levi factor of $\phi(G)^{*_{0}}$. Then $\phi(N) S$ is a normal subgroup of $\phi(G)^{*_{0}}$, since $\phi(N)$ is the representation radical of $\phi(G)^{*_{0}}$. It is clear that $\phi\left(D_{\lambda}\right)$ is not a subgroup of $\phi(N) S$ for almost all $\lambda \in \Lambda$. Now, $\phi(G)^{*} / \phi(N) S$ is a compactly generated abelian group. Let $F=\phi^{-1}(\phi(N) S)$. Then $G / F$ is a locally compact abelian group and it is compactly generated since $G$ is. By the structure theorem of compactly generated locally compact abelian groups, $G_{1} F / F$ can contain an infinite torsion group. (Observe that $G_{0} F / F$ is the connected component of the abelian group $G / F$.) Therefore $G_{1} / G_{0}$ is finite. The proof is complete.

Recall the following result (Corollary 4.11 of [19]): Let $G$ be a finitely generated subgroup of $\mathrm{GL}(n, F)$, where $F$ is a field. Then every completely reducible solvable subgroup of $G$ is finitely generated. Now, assume $\phi\left(G_{0}\right)$ is almost algebraic, i.e., $\phi\left(G_{0}\right)$ has finite index in $\phi\left(G_{0}\right)^{*}$. If $G_{1} / G_{0}$ is not finite, then $\bigcup_{\lambda \in \Lambda} V D_{\lambda}$ is not finite. Furthermore $\phi\left(G_{0}\right)^{*} \phi\left(D_{\lambda}\right) / \phi\left(G_{0}\right)^{*}$ is normal in $\phi(G)^{*} 0 / \phi\left(G_{0}\right)^{*}$, so except for finitely many indices, $\phi\left(D_{\lambda}\right)$ is contained in the radical of $\phi(G)^{*}$ and it is soluble. Now, if $G$ is compactly generated, then $G$ is generated by a finite subset $\left\{x_{1}, \ldots, x_{n}\right\}$ of $G$ and $G_{0}$, so $\phi(G)$ is generated by $\left\{\phi\left(x_{1}\right), \ldots, \phi\left(x_{n}\right)\right\}$ and $\phi\left(G_{0}\right)$. Extending the base field to the complex number field and taking the quotient over $\phi\left(G_{0}\right)_{\mathbf{C}}$ we see that $\phi\left(V D_{\lambda}\right)$ is finite by the above mentioned result. Thus we have the following condition:

16. Proposition. Let $G$ be a locally generated group with trivial maximal normal subgroup. Let $\phi$ be the adjoint representation of $G$ on the Lie algebra $\mathscr{L}\left(G_{0}\right)$ of $G_{0}, \phi: G \rightarrow \operatorname{GL}\left(\mathscr{L}\left(G_{0}\right)\right)$. If $\phi\left(G_{0}\right)$ is almost algebraic, i.e., $\phi\left(G_{0}\right)$ has finite index in $\phi\left(G_{0}\right)^{*}$, then $G / G_{0}$ is an $(\mathrm{H})$ group. 
We note that when $G_{1} / G_{0}$ is not finite, $G_{0}$ is not almost algebraic. Let $\Gamma$ denote the subgroup generated by $\left\{\phi\left(x_{1}\right), \ldots, \phi\left(x_{n}\right)\right\}$. Then there are infinitely many elements from $V D_{\lambda}$ which are in $\phi\left(G_{0}\right)^{*}$ but not in $\phi\left(G_{0}\right)$, and they have the form $d=y \gamma$, where $d \in \phi\left(G_{0}\right)^{*}, y \in \phi\left(G_{0}\right)$, and $\gamma \in \Gamma$. This situation is illustrated in Example 21.

17. Example. (A compactly generated solvable group which is not an $(\mathrm{H})$ group.) Let $G$ be the semidirect product: $Z{ }_{\eta} \sum_{i=-1}^{-\infty}\left(Z_{2}\right) i \times \prod_{j=0}^{\infty}\left(Z_{2}\right)_{j}$, where $-\infty<i \leq-1,0 \leq j<\infty$ with $\sum Z_{2}$ discrete and $\prod Z_{2}$ the product topology. The action $\eta$ is the shifting of the indices.

18. Example. (A finitely generated discrete solvable group $G$, where $\mathscr{D}(G)$ is not finitely generated and also the center of $G$ is not finitely generated.) We sketch the example and omit the details $[19,4.21]$. The group $G$ is a subgroup of $\mathrm{GL}(3, \mathbf{R})$ generated by the following matrices:

$$
a=\left(\begin{array}{lll}
1 & 1 & 0 \\
0 & 1 & 0 \\
0 & 0 & 1
\end{array}\right), \quad b=\left(\begin{array}{ccc}
1 & 0 & 0 \\
0 & 1 & 1 \\
0 & 0 & 1
\end{array}\right), \quad t=\left(\begin{array}{ccc}
1 & 0 & 0 \\
0 & 1 / 2 & 0 \\
0 & 0 & 1
\end{array}\right) .
$$

The center of $G$ consists of matrices

$$
\left(\begin{array}{ccc}
1 & 0 & \sum m_{i}(1 / 2)^{i} \\
0 & 1 & 0 \\
0 & 0 & 1
\end{array}\right)
$$

where the $m_{i}$ are integers and the center is not finitely generated. Observe that $D^{(1)}(G)$ is not finitely generated. It is easy to check that $D^{(1)}(G) / D^{(2)}(G)$ is not a subgroup of $B\left(G / D^{(2)}(G)\right)$.

19. Example. (A totally disconnected compactly generated locally compact group which has a uniform solvable subgroup but it is not an (H) group.) Consider those semisimple $p$-adic Lie groups with respect to Iwasawa decompositions [20].

So far, we have seen the importance of the assumption that $G$ is compactly generated. The following example shows what can happen when the group is not compactly generated.

20. Example. Let $G_{i}=K_{i} \cdot H_{i}$ be the semidirect product, where $K_{i}$ is a finite subgroup and $H_{i}$ is a finitely generated discrete FC group, $i=1,2, \ldots$ Let $G$ be the local direct product of the family $\left\{\left(G_{i}, K_{i}\right): i=1,2, \ldots\right\}$. If $K_{i}$ is a nontrivial group of automorphisms of $H_{i}$ for each $i \in Z^{+}$, then $G$ is not compactly generated and there are no compact-open normal subgroups of $G$.

21. Example. (The group $G$ is a compactly generated, $(\mathrm{H})$ group but $G / G_{0}$ is not an $(\mathrm{H})$ group.) This example is a combination of the example in Remark 12 and Example 18.

Let $H=S \cdot \mathrm{C}$, with $S$ the subgroup of nonzero complex numbers of module one and $\mathbf{C}$ is the additive group of complex numbers. Let $E$ be the subgroup 
of $\mathrm{GL}(3, \mathbf{R})$ generated by the matrices $a, b, t$ and

$$
\left(\begin{array}{lll}
1 & 0 & r \\
0 & 1 & 0 \\
0 & 0 & 1
\end{array}\right): r \in \mathbf{R}
$$

here $a, b, t$ have the same meaning as in Example 18. Let $F$ be the semidirect product $E \cdot \mathbf{R}^{3}$. Here $E$ acts on $\mathbf{R}^{3}$ as a subgroup of $\operatorname{GL}(3, \mathbf{R})$. The group $H \times F$ is the semidirect product $(S \times E) \cdot\left(\mathbf{C} \times \mathbf{R}^{3}\right)$. The group $G$ which we shall construct is a subgroup of $H \times F$ obtained from $H \times F$ by taking a subgroup of $S \times E$. Let $P$ be the one-parameter subgroup of $S \times E$ :

$$
P=\left\{\left(e^{2 \pi r \sqrt{-1}},\left(\begin{array}{lll}
1 & 0 & r \\
0 & 1 & 0 \\
0 & 0 & 1
\end{array}\right)\right): r \in \mathbf{R}\right\}
$$

Let $E^{\prime} \subset S \times E$ be generated by $(1, a),(1, b),(1, t)$, and let $Q$ be the subgroup generated by $P$ and $E^{\prime}$. Then $G=Q \cdot\left(\mathbf{C} \times \mathbf{R}^{3}\right)$. It is clear that $P \cdot\left(\mathbf{C} \times \mathbf{R}^{3}\right)$ is an analytic group under the natural Euclidean topology. Now, define the topology of $G$ by taking $P \cdot\left(\mathbf{C} \times \mathbf{R}^{3}\right)$ as an open subgroup, i.e., $G_{0}=P \cdot\left(\mathbf{C} \times \mathbf{R}^{3}\right)$. Then $G$ is a compactly generated $(\mathbf{H})$ group. To see that $G / G_{0}$ is not an $(\mathrm{H})$ group, one simply observes that

$$
\left\{\left(e^{2^{n} \pi \sqrt{-1}},\left(\begin{array}{lll}
1 & 0 & 0 \\
0 & 1 & 0 \\
0 & 0 & 1
\end{array}\right)\right): n \in Z\right\} \subset Q .
$$

Therefore $G / G_{0}$ is not an $(\mathrm{H})$ group.

22. Weakly boundedness. We have seen that the boundedness condition plays an important role in the structure of locally compact groups. In the second half of this paper (after Proposition 4) we used this condition critically. In fact, the first half (i.e., Proposition 4) could be done by a weakened notion of boundedness. Let $x$ be an element of the locally compact group $G$. Let $N(x)$ be the smallest closed normal subgroup of $G$ which contains $x$. Observe when $x \in B(G), N(x)$ satisfies the following conditions: (1) $N(x)$ is an (H) group and (2) every closed subgroup of $N(x)$ is compactly generated. Now, we use these two conditions to define weak boundedness. We say that $x$ is weakly bounded if and only if $N(x)$ is an $(\mathrm{H})$ group and every closed subgroup of $N(x)$ is compactly generated. Let $B^{*}(G)$ denote the subset of $G$ consisting of all the weakly bounded elements. We are going to show that $B^{*}(G)$ could be used to develop a similar theory to that of $B(G)$.

In the following, we shall always assume that $G$ is a totally disconnected locally compact group.

23. Lemma. Let $G$ be a totally disconnected locally compact group. Then $B^{*}(G)$ is a group.

Proof. Let $x$ and $y$ be any two elements of $B^{*}(G)$. Let $K(x)$ and $K(y)$ denote the maximal compact normal subgroups of $N(x)$ and $N(y)$, respectively. 
Since $K(x)$ is a characteristic subgroup of $N(x)$ and $N(x)$ is normal in $G$, it follows that $K(x)$ is normal in $G$. Similarly $K(y)$ is normal in $G$.

Let $F=(N(x) N(y))^{-}$. We are going to show that $F$ is an (H) group. Without loss of generality, we can assume that $N(x)$ and $N(y)$ both are discrete subgroups, since we can factor out $K(x) K(y)$ from $G$. Let $D^{\prime}$ be any compactopen subgroup of $F$. Since $N(x)$ is discrete and finitely generated, there exists an open subgroup $D_{1}$ of $D^{\prime}$ which centralizes $N(x)$. Similarly, there exists an open subgroup $D_{2}$ of $D^{\prime}$ which centralizes $N(y)$. Therefore, $D=D_{1} \cap D_{2}$ is in the center of $F$. Hence $F=N(x) N(y) D$, where $D$ is a compact open central subgroup of $F$. Observe that $F$ is compactly generated. We wish to show that every closed subgroup of $F$ is compactly generated. For this purpose, we need to make a detour first. Let $(N(x) N(y))_{d}$ denote the group $N(x) N(y)$ with discrete topology. Then $(N(x) N(y))_{d}$ is a countable group (since it is finitely generated). We have an isomorphism from $(N(x) N(y))_{d} / E \rightarrow N(x) N(y) D / D$, where $E$ is a subgroup of $(N(x) N(y))_{d}$ and $E$ is the kernel of the inclusion map $(N(x) N(y))_{d} \rightarrow N(x) N(y) D, E=D \cap N(x) N(y)$. Now, we wish to show that every subgroup of $(N(x) N(y))_{d}$ is finitely generated. Let $L$ be any subgroup of $(N(x) N(y))_{d}$. In the following, we shall write $N(x) N(y)$ for $(N(x) N(y))_{d}$. Observe that $L N(y) / N(y)$ is isomorphic to a subgroup of $N(x) N(y) / N(y)$. We have $N(x) N(y) / N(y) \cong N(x) / N(x) \cap N(y)$. Since every subgroup of $N(x)$ is finitely generated, $L N(y) / N(y)$ is finitely generated. On the other hand, $L / L \cap N(y)$ is isomorphic to $L N(y) / N(y)$. Since every subgroup of $N(y)$ is finitely generated, $L \cap N(y)$ is finitely generated. Therefore, $L$ is finitely generated.

Now, we can prove that every closed subgroup $E$ of $F$ is compactly generated. Since $E D / D$ is isomorphic to $E / E \cap D, E$ is compactly generated if and only if $E D / D$ is finitely generated. Since $E D / D$ is isomorphic to a subgroup of $(N(x) N(y))_{d}, E D / D$ is finitely generated, a fortiori, $E$ is compactly generated. In particular, the center $Z(F)$ of $F$ is compactly generated. By the structure theorem of compactly generated locally compact abelian groups, $Z(F)=Z^{l} \times K$, where $K$ is a maximal compact subgroup of $Z(F)$. Since $Z(F) \supset D, K \supset D$. Now, it is clear that $F$ is an $(\mathrm{H})$ group. Furthermore, every closed subgroup of $F$ is compactly generated. Since $x y \in F, x y \in B^{*}(G)$ and $B^{*}(G)$ is a group.

24. Examples. (a) Let $G=Z{ }_{\eta} Z^{2}$, where $\eta$ is defined by the matrix $\left(\begin{array}{ll}1 & 1 \\ 3 & 4\end{array}\right)$. Then $B^{*}(G)$ properly contains $B(G)$.

(b) Let $G=\left(Z_{2}\right){ }_{\eta} Z$, where $\eta(n)=-n$. Then $G=B^{*}(G)$, but $P(G) \subset$ $B(G)$. Unlike the known fact that $P(G) \cap B(G)$ is a group, this example shows that $P(G) \cap B^{*}(G)$ is not a group.

25. Proposition. Let $G$ be a totally disconnected locally compact group and let $F$ be a compactly generated closed normal subgroup of $G$. Assume that $F \subset B^{*}(G)$. Then $F$ is an $(\mathrm{H})$ group and every closed subgroup of $F$ is compactly generated. 
Proof. Since $F$ is compactly generated, it is generated by a finite subset $x_{1}$, $\ldots, x_{n}$ of $F$ and a compact open subgroup $K$ of $F$. Let $N_{i}=N\left(x_{i}\right)$ and $K_{i}=K\left(x_{i}\right)$ for $i=1, \ldots, n$. Then $F=\left(\prod_{i=1}^{n} N_{i}\right)^{-} K$. Let $E=\left(\prod_{i=1}^{n} N_{i}\right)^{-}$. By Lemma 23 (and finite induction), $E$ is an $(\mathrm{H})$ group and every closed subgroup is compactly generated. So $E$ has a maximal compact normal subgroup $J$. Let $\theta$ be the quotient homomorphism $G \rightarrow G / J$. Let $E^{\prime}=\theta(E)$, $F^{\prime}=\theta(F)$, and $K^{\prime}=\theta(K)$. Then $E^{\prime}$ is a finitely generated discrete group normal in $G^{\prime}$. Now, using the representation of $K^{\prime}$ on $E^{\prime}$ by inner automorphisms, it is clear that there is an open subgroup $M^{\prime}$ of $K^{\prime}$ which centralizes $E^{\prime}$. Following the same type argument as in the proof of Lemma 23, we see that $F$ is an $(\mathrm{H})$ group and every closed subgroup is compactly generated.

26. Proposition. Let $H$ be a totally disconnected locally compact group. Assume that $H$ has a finite sequence of closed normal subgroups: $H=H_{1} \supset H_{2} \supset$ $\cdots \supset H_{n} \supset H_{n+1}=(e)$ such that (1) $H_{i} / H_{i+1}$ is compactly generated and (2) $H_{i} / H_{i+1} \subset B^{*}\left(H / H_{i+1}\right)$ for $1 \leq i \leq n$. Then $H$ is an $(\mathrm{H})$ group and every closed subgroup of $H$ is compactly generated.

Proof. First, we show that $H$ itself is an $(\mathrm{H})$ group. The proof follows the same line as that of Lemma 2. We supply here a few details which are needed since $H_{i}$ now may not be an abelian group. We use induction on the length of the series $H=H_{1} \supset \cdots \supset H_{n}$. Without loss of generality, we assume that $H_{n}$ is discrete and finitely generated. We assume $H / H_{n}$ is an $(\mathrm{H})$ group. This implies there exists a normal subgroup $M$ of $H$ such that (1) $H_{n} \subset M \subset H, H / M$ is a discrete group such that every subgroup of $H / M$ is finitely generated, and (2) $M / H_{n}$ is compact. Let $\phi$ be the representation of $M$ on $H_{n}$, defined by inner automorphisms, $\phi: M \rightarrow$ Aut $H_{n}$. Since $H_{n}$ is discrete, Aut $H_{n}$ is also discrete. Let $Z_{M}\left(H_{n}\right)$ be the centralizer of $H_{n}$ in $M$. Then $Z_{M}\left(H_{n}\right) H_{n}=$ $\phi^{-1} \phi\left(H_{n}\right)$. Since $\phi\left(H_{n}\right)$ is discrete, it is closed. Hence $Z_{M}\left(H_{n}\right) H_{n}$ is a closed subgroup. Since $\phi(E) / \phi\left(H_{n}\right)$ is compact, it is finite. Therefore, $Z_{M}\left(H_{n}\right) H_{n}$ is an open subgroup with finite index. Since $Z_{M}\left(H_{n}\right) / Z_{M}\left(H_{n}\right) \cap H_{n}$ is isomorphic to $Z_{M}\left(H_{n}\right) H_{n} / H_{n}, Z_{M}\left(H_{n}\right) \cap H_{n}$ is uniform in $Z_{M}\left(H_{n}\right)$. Certainly $Z_{M}\left(H_{n}\right) \cap$ $H_{n}$ is central in $Z_{M}\left(H_{n}\right)$. Hence $Z_{M}\left(H_{n}\right)$ is a compactly generated $(Z)$ group. Let $D$ be the maximal compact normal subgroup of $Z_{M}\left(H_{n}\right)$. Then $D$ is open in $M$, a fortiori, it is open in $H$. Therefore, $H$ is an $(\mathrm{H})$ group. It follows by the same argument as that of Lemma 2, that every subgroup of $H$ is compactly generated.

27. Remarks. (1) In the definition of weak boundedness, we require that every closed subgroup be compactly generated. This condition is needed, as seen from the above proof, when we assert that $M$ is a compactly generated $(Z)$ group.

(2) Let $G$ be a locally compact topological group and let $H$ be a uniform normal subgroup of $G$. Assume $H$ satisfies the condition of Proposition 26. Then one can prove that $G$ is an (H) group (cf. [9]). 
We have seen that $P(G) \cap B^{*}(G)$ is not contained in $B(G)$, i.e., a compact weakly bounded element may not be a bounded element, and also that $P(G) \cap$ $B^{*}(G)$ may not be a subgroup (Example 24(b)). However, $P(G) \cap B^{*}(G)$ is not too far away from $B(G)$ as the following Proposition 29 shows.

28. Lemma. Let $x \in P(G) \cap B^{*}(G)$ and $N=N(x)$. Then either $x \in B(G)$ or $x \notin B(N)$.

Proof. Let $x \in P(G) \cap B^{*}(G)$, and let $N=N(x)$ be the smallest closed normal subgroup of $G$ which contains $x$. If $x \in B(N)$, i.e., $x$ is bounded as an element in $N$, then $x \in B(N) \cap P(N)$, a fortiori, $x \in K(x)$. This implies that $x \in K(x)=N(x)$, and $x \in B(G) \cap P(G)$. Hence a compact weakly bounded $x$ is either a bounded element in $G$ or it is not bounded in $N=N(x)$.

Let $X$ be a subset of $G$ and let $N(X)$ be the smallest closed normal subgroup of $G$ which contains $X$. When $X=\left\{x_{1}, \ldots, x_{m}\right\}$, then $N(X)=$ $\left(N\left(x_{1}\right) \cdots N\left(x_{m}\right)\right)^{-}$.

30. Proposition. Let $D$ be a compact subgroup of $B^{*}(G)$. Then there exists a finite subset $d_{1}, \ldots, d_{s}$ of $D$ such that $\theta(D) \subset B(\theta(G))$, where $\theta: G \rightarrow$ $G / N\left(d_{1}, \ldots, d_{s}\right)$.

Proof. By the proof of Lemma $23, N\left(d_{1}, \ldots, d_{m}\right)$ is an $(\mathrm{H})$ group. Let $K\left(d_{1}, \ldots, d_{m}\right)$ be the maximal compact normal subgroup of $N\left(d_{1}, \ldots, d_{m}\right)$. Then $N\left(d_{1}, \ldots, d_{m}\right) / K\left(d_{1}, \ldots, d_{m}\right)$ is a discrete finitely generated group. The automorphism group

$$
\operatorname{Aut}\left(N\left(d_{1}, \ldots, d_{m}\right) / K\left(d_{1}, \ldots, d_{m}\right)\right)
$$

is discrete. Therefore, there exists a subgroup of $D$ of finite index which centralizes $N\left(d_{1}, \ldots, d_{m}\right) / K\left(d_{1}, \ldots, d_{m}\right)$. Now, we wish to show that there exists a finite subset $\left\{d_{1}, \ldots, d_{s}\right\}$ such that every element $d$ in $D$ is a bounded element in $G / N\left(d_{1}, \ldots, d_{s}\right)$. We prove this by contradiction. Hence we assume for any finite subset $\left\{d_{1}, \ldots, d_{m}\right\}$ of $D$, there exists an element $d_{m+1}$ such that $d_{m+1}$ is not bounded in $G / N\left(d_{1}, \ldots, d_{m}\right)$, i.e.,

$$
N\left(d_{m+1}\right) N\left(d_{1}, \ldots, d_{m}\right) / N\left(d_{1}, \ldots, d_{m}\right)
$$

is not compact. Let $d_{1} \in D$ and $d_{1} \notin B(G)$. There is an open subgroup $D_{1}$ of $D$ such that $D_{1}$ centralizes $N\left(d_{1}\right) / K\left(d_{1}\right)$. Since $D / D_{1}$ is finite, it has a finite set of representatives of $D$ over $D_{1}$. If every element $d$ in $D_{1}$ is a bounded element in $G / N\left(D_{1}\right)$, then every element $d$ in $D$ is a bounded element in $G / N\left(d_{1}\right)$. Therefore, there exists at least an element $d_{2}$ in $D_{1}$ such that $d_{2}$ is not bounded in $G / N\left(d_{1}\right)$.

Now, let $\phi$ be the representation of $G$ on $N\left(d_{1}\right) / K\left(d_{1}\right)$ defined by the inner automorphisms. Then $D_{1}$ is contained in the kernel of $\phi$. Since the kernel of $\phi$ is a normal subgroup, and $d_{2} \in$ kernel of $\phi$, then $N\left(d_{2}\right)$ is a subgroup of the kernel of $\phi$. Therefore, for any $n_{1} \in N\left(d_{1}\right), n_{2} \in N\left(d_{2}\right)$, we have 
$n_{2} n_{1} d_{1} n_{1}^{-1} n_{2}^{-1}=n_{1} d_{1} n_{1}^{-1} k_{1}$ for some $k_{1} \in K\left(d_{1}\right)$, and $n_{2} n_{1} d_{1} d_{2} n_{1}^{-1} n_{2}^{-1}=$ $\left(n_{1} d_{1} n_{1}^{-1}\right)\left(n_{2} d_{2} n_{2}^{-1}\right) k_{1}^{\prime}$ for some $k_{1}^{\prime} \in K\left(d_{1}\right)$.

Now, suppose we have defined $d_{1}, \ldots, d_{m}$ with the properties: (1) $d_{j+1} \in$ $D_{j}$, where $D_{j}$ is an open subgroup of $D$ which centralizes

$$
N\left(d_{1}\right) / K\left(d_{1}\right), \ldots, N\left(d_{j-1}\right) / K\left(d_{j-1}\right),
$$

and (2) the image of $d_{j+1}$ is not a bounded element in $G / N\left(d_{1}, \ldots, d_{j}\right)$ for $2 \leq j+1 \leq m$. It is clear we can define $d_{m+1}$ with the same properties. Therefore, we have a sequence $d_{1}, d_{1} d_{2}, d_{1} d_{2} d_{3}, \ldots$ in $D$. Since $D$ is compact, there exists a subnet of the above sequence which converges to a point $d \in D$. Since $D \subset B^{*}(G)$ (factoring out $K(d)$ ), without loss of generality, we may assume that $N(d)$ is discrete. So every subgroup of $N(d)$ is finitely generated by the definition of weakly bounded element.

Let $F$ be the subgroup of $N(d)$ generated by $\left\{n_{1} n_{2} \cdots n_{l} d n_{l}^{-1} \cdots n_{1}^{-1}: n_{i} \in\right.$ $N\left(d_{i}\right)$ and $l$ is any positive integer $\}$. Observe $d=d_{1} d_{2} \cdots d_{l} d_{l}^{\prime}$, where $d_{l}^{\prime}=$ $d_{l}^{-1} d_{l-1}^{-1} \cdots d_{1}^{-1} d$ and $d_{l}^{\prime}$ is the limit of a subnet of

$$
\left\{d_{l+1}, d_{l+1} d_{l+2}, d_{l+1} d_{l+2} d_{l+3}, \ldots\right\},
$$

and we have

$$
n_{1} n_{2} \cdots n_{l} d n_{l}^{-1} \cdots n_{1}^{-1}=n_{1} d_{1} n_{1}^{-1} n_{2} d_{2} n_{2}^{-1} \cdots n_{l} d_{l} n_{l}^{-1} d_{l}^{\prime} k_{1} k_{2} \cdots k_{l},
$$

where $k_{i} \in K\left(d_{i}\right), 1 \leq i \leq l$. Then $F$ is not a finitely generated group. This contradicts the fact that every subgroup of $N(d)$ is finitely generated. Therefore, we conclude there exists a finite subset $d_{1}, \ldots, d_{s}$ of $D$ such that $\theta(D) \subset B\left(G / N\left(d_{1}, \ldots, d_{s}\right)\right)$. Now, the proof is complete.

31. Proposition. Let $D$ be a compact subgroup of $B^{*}(G)$. Then there exists an open subgroup $D^{\prime}$ of $D$ such that $D^{\prime} \subset B(G)$.

Proof. By Proposition 30, there exists $N=N\left(d_{1}, \ldots, d_{s}\right)$ such that $\pi(D) \subset$ $B(\pi(G))$, where $\pi$ is the homomorphism from $G$ onto $G / N$. By Proposition 38 (whose proof is independent from that of the present proposition), $N(\pi(D)$ ) is a compact normal subgroup of $\pi(G)$. Let $F=\pi^{-11}(N(\pi(D)))$. Then $F$ is normal in $G$ and $N$ is uniform in $F$. By the proof of Proposition 26, $F$ has a maximal compact normal subgroup $M$ such that $F / M$ is discrete, i.e., $M$ is open in $F$. It is clear that $M \subset B(G)$. Since $M$ is open in $F, D^{\prime}=F \cap D$ is open in $D$ and the proof is complete.

33. Corollary. Let $G$ be a totally disconnected locally compact group with $B^{*}(G)$ an open subgroup. Let $D$ be a compact subset of $B^{*}(G)$. Then $N(D)$ is compactly generated. If $D$ is a compact subset of $P(G) \cap B(G)$, then $N(D)$ is compact.

Proof. Let $K$ be a compact-open normal subgroup of $G$. Let $\theta$ be the quotient homomorphism from $G$ onto $G / K$. Since $G / K$ is discrete, $\theta(D)$ is finite 
and $N(\theta(D))$ is a finitely generated normal subgroup. Therefore, $N(D)=$ $\theta^{-1}(N(\theta(D)))$ is compactly generated. When $D \subset B(G) \cap P(G), N(\theta(D))$ is a finite group, so $N(D)$ is a compact normal subgroup.

34. Let $D$ be a compact subset of $P(G) \cap B(G)$. In general, the smallest closed normal subgroup $N(D)$ of $G$ which contains $D$ is not compact. As an example: Let $G=\prod_{i=1}^{\infty}\left(Z_{2}\right)_{i} \cdot \sum\left(Z_{3}\right)_{i}$; here $\left(Z_{2}\right)_{i}$ is identified as the group of automorphisms of $\left(Z_{3}\right)_{i}$ which is a copy of $Z_{3}$. Let $D$ be the subset of $\prod_{i=1}^{\infty}\left(Z_{2}\right)_{i}$ which has at most one nontrivial component. The closed subgroup generated by $D$ is $\prod_{i=1}^{\infty}\left(Z_{2}\right)_{i}$ and the smallest closed normal subgroup $N(D)$ which contains $D$ is the group $G$. So it is not compactly generated.

35. Proposition. Let $G$ be a locally compact totally disconnected group, let $D$ be a compact subset of $B(G) \cap P(G)$, and let $N(D)$ be the smallest closed normal subgroup of $G$ which contains $D$. Then $N(D)$ is compact if and only if $N(D) \subset B(G)$.

Proof. (1) If $N(D)$ is compact, then $N(D)$ is a subgroup of $B(G)$. (2) Assume that $N(d)$ is a subgroup of $B(G)$. Let $G^{\prime}$ be the locally compact group obtained from $G$ by adding $N(D)$ as an open subset to the original topology of $G$. Let $\theta$ be the identification map from $G^{\prime}$ to $G$. We shall write $x^{\prime}$ for $\theta^{-1}(x)$ and $E^{\prime}=\theta^{-1}(E)$ for a point $x$ or subset $E$ of $G$. Then $G^{\prime}$ is a locally compact totally disconnected group. Since $N\left(D^{\prime}\right) \subset B\left(G^{\prime}\right)$, it follows that $B\left(G^{\prime}\right)$ is open. By Theorem 1 of [16], $G^{\prime}$ has a compact normal open subgroup $K^{\prime}$. Observe that $N\left(D^{\prime}\right)$ is topologically isomorphic with $N(D)$. Now $D$ is compact in $G$, so $D^{\prime}$ is compact in $G^{\prime}$. Thus, $D^{\prime} \subset \bigcup_{j=1}^{m} d_{j}^{\prime} K^{\prime}$, where $d_{j}^{\prime} \in D^{\prime}$, and

$$
D \subset \bigcup_{j=1}^{m} d_{j} K \subset \prod_{j=1}^{m} N\left(d_{j}\right) K .
$$

Since $\prod_{j=1}^{m} N\left(d_{j}\right) K$ is a compact normal subgroup of $G$, a fortiori, $N(D)=$ $\prod_{j=1}^{m} N\left(d_{j}\right) K$ and $N(D)$ is compact. Now, the proof is complete.

36. Corollary. Let $G$ be a totally disconnected locally compact group. Let $D$ be a compact subset of $B(K) \cap P(G)$. If $B(G)$ is closed, then $N(D)$ is a compact subgroup.

37. Proposition. Let $G$ be a locally compact totally disconnected group and let $D$ be a compact subset of $B(G)$. If $N(D) \subset B(G)$, then $N(D)$ is compactly generated.

Proof. We only need to make the following changes from the proof of Proposition 35. Since $D \subset \prod_{i=1}^{m} N\left(d_{i}\right) K=N(D)$ and each $N\left(d_{i}\right)$ is compactly generated, $N(D)$ is compactly generated.

38. Proposition. Let $G$ be a totally disconnected locally compact group and let $D$ be a compact subgroup of $B(G)$. Then the smallest closed normal subgroup of $G$ which contains $D$ is compact. 
Proof. Let $N(D)$ be the smallest closed normal subgroup of $G$ which contains $D$. Without loss of generality, we may assume that $N(D)$ is an open subgroup (cf. the proof of Proposition 35). Let $\mathscr{N}(D)$ be the subgroup generated by $\{N(d): d \in D\}$. Then $\mathscr{N}(D)$ is a dense subgroup of $N(D)$. Let $L$ be any compact open subgroup of $N(D)$. Then $\mathscr{N}(D) L=N(D)$. If $N(D)$ is not compact, then $N(D) / L$ is an infinite discrete space. We shall prove our assertion by contradiction, so we assume $N(D)$ is not compact. Let $d_{1}$ be any element in $D$ such that $d_{1}^{G}$ is not completely contained in $L$; such an element $d_{1} \in D$ exists, otherwise $D^{G} \subset L$ and $N(D)$ is compact. Let $f_{1} \in G$ such that $f_{1} d_{1} f_{1}^{-1} \notin L$. Let $D_{1}$ be an open compact subgroup of $D$ such that $D_{1} \subset L$ and $f_{1} D_{1} f_{1}^{-1} \subset L$. Now, $N\left(D_{1}\right) L$ is compact, so there exists an element $f_{2} \in G$ and $d_{2} \in D_{1}$ such that $f_{2} d_{2} f_{2}^{-1} \in N\left(d_{1}\right) L$. Observe that

$$
f_{2} d_{1} d_{2} f_{2}^{-1}=\left(f_{2} d_{1} f_{2}^{-1}\right)\left(f_{2} d_{2} f_{2}^{-1}\right) \in\left(f_{2} d_{2} f_{2}^{-1}\right) N\left(d_{1}\right) L,
$$

and

$$
f_{1} d_{1} d_{2} f_{1}^{-1}=\left(f_{1} d_{1} f_{1}^{-1} \lambda f_{1} d_{2} f_{1}^{-1}\right) \in\left(f_{1} d_{1} f_{1}^{-1}\right) L .
$$

So, $\left(f_{2} d_{1} d_{2} f_{2}^{-1}\right) L$ and $\left(f_{1} d_{1} d_{2} f_{1}^{-1}\right) L$ are in different cosets and both are different from $L$. Now, we can find an open compact subgroup $D_{2}$ of $D$ such that $D_{2} \cup f_{1} D_{2} f_{1}^{-1} \cup f_{2} D_{2} f_{2}^{-1} \subset L$. Choose $f_{3} \in G$ and $d_{3} \in D_{3}$ so that $f_{3} d_{3} f_{3}^{-1}$ does not belong to $N\left(d_{1}\right) N\left(d_{2}\right) L$.

It is clear that this process can be continued indefinitely and we obtain a sequence of elements from $D:\left\{d_{1}, d_{1} d_{2}, d_{1} d_{2} d_{3}, \ldots\right\}$. Since $D$ is compact, there is a subnet of the above sequence which converges to an element $d \in D$. It is clear that the orbit $d^{G}$ of $d$ contains an infinite discrete subset $f_{1} d f_{1}^{-1}, f_{2} d f_{2}^{-1}, \ldots$ This violates the condition that $N(d)$ is compact. Therefore $N(D)$ must be compact, and the proof is now complete.

39. Corollary. Let $G$ be a totally disconnected locally compact group. Then $B(G)$ is closed if and only if $B(G) \cap P(G)$ is closed in $G$.

Proof. If $B(G)$ is closed, $\overline{P(G) \cap B(G)} \subset B(G)$. By Lemma 1 of [16, §3], $P(G) \cap B(G)$ is the union of a compact open subgroup of $G$, so $\overline{P(G) \cap B(G)} \subset$ $P(G)$. Therefore, $(P(G) \cap B(G))^{-} \subset P(G) \cap B(G)$, so $P(G) \cap B(G)$ is closed.

Conversely, assume $P(G) \cap B(G)$ is closed in $G$, and let $K$ be a compactopen subgroup of $G$. Since $K \subset P(G), D=K \cap B(G)=K \cap(P(G) \cap B(G))$, where $D$ is a compact-open subgroup of $B(G)$. So $N(D)$ is a compact open subgroup of $B(G)$. Therefore $B(G)$ is locally compact, and $B(G)$ is closed in $G$.

40. Remark. Let $H$ be a totally disconnected locally compact topological group. If $H=B(H)$ and $H$ is compactly generated, then $H=B(H)$ [16]. So if either $(B(G) \cap P(G))^{-}$or $B(G)^{-}$is compactly generated, then $B(G)$ is closed.

41. Proposition. Let $G$ be a $\sigma$-compact totally disconnected locally compact group. If $B^{*}(G)$ is closed, then $B(G)$ is closed. 
Proof. We use the same method as in the proof of Proposition 35. Let $G^{\prime}$ be the locally compact group obtained from $G$ by adding $B^{*}(G)$ as an open subset. Then $G^{\prime}$ has a compact-open invariant subgroup. So $B\left(G^{\prime}\right)$ is open in closed in $G^{\prime}$. Observe that $\theta^{-1}(B(G))$ is an open subgroup in $G^{\prime}$ since it contains the invariant compact open subgroup of $G^{\prime}$. Therefore, let $\theta$ be the identification map from $G^{\prime}$ onto. Since $G$ is $\sigma$-compact, so is $B^{*}(G)$. Therefore, $\theta \mid \theta^{-1}\left(B^{*}(G)\right)$ is a homeomorphism. Hence $B(G)$ is locally compact, and $B(G)$ is closed in $G$.

42. Questions. Assume $G$ is totally disconnected.

(1) Let $D$ be a closed normal subset of $G$. If $D \subset B(G)$, is $N(D) \subset B(G)$ ? ( $D$ is normal if $D^{G}=D$.)

(2) Let $D \subset B(G)$. Let $\mathscr{N}(D)$ denote the smallest normal subgroup of $G$ which contains $D$. Then $N(D)=\mathscr{N}(D)^{-}$. If $N(D)$ is compactly generated, is $\mathscr{N}(D)$ also compactly generated?

(3) Let $H$ be a closed subgroup of $G$ such that $G / H$ admits a finite left invariant measure. Is $H$ uniform in $G$ ?

(4) Let $D \subset B(G)$-we say $D$ is uniformly bounded if $D^{G}$ is relatively compact. Are there any reasonable conditions on $D$ so that $D$ is uniformly bounded?

(5) When does there exist a collection of compactly generated open normal subgroups whose intersection is the identity or a compact subgroup of $G$ ? (Cf. Example 17.)

(6) Study $B^{*}(G)$ when $G$ is not totally disconnected.

(7) When $N(x)$ is finite for each $x \in P(G) \cap B(G)$, what additional information may we possibly obtain from this condition?

(8) Let $G$ be a compactly generated locally compact totally disconnected solvable group. Assume that $D^{(1)}(G)$ is abelian and $B(G) \cap D^{(1)}(G)$ is dense in $D^{(1)}(G)$. Is $D^{(1)}(G)$ compactly generated?

(9) Let $G$ be a locally compact totally disconnected group. Let $\theta$ be an automorphism of $G$. Assume there exists a dense orbit of $G$ (i.e., $\left\{\theta^{n}(x) \mid n \in Z\right\}^{-}$ $=G)$. Is $G$ compact?

(10) Let $G$ be an abelian totally disconnected locally compact group. Let $\theta$ be an automorphism of $G$. Assume there exists a compact open subgroup $K$ of $G$ such that $G$ is generated by $K$ and $\theta$, i.e., $G$ is generated by $\left\{\theta^{n}(k): n \in\right.$ $Z$ \}. What is the structure of $G$ ?

(11) Find a suitable definition to characterize "smallness" in totally disconnected locally compact groups. Let $G$ be a locally compact group. Find the conditions so that $G=H K$, where $K$ is an open compact subgroup and $H \cap K$ is small. (Say $\mu(H \cap K)=0$, where $\mu$ is a Haar measure on $K$.) Let $\mathscr{B}=\{H: G=H K\}$. Study the conjugacy classes of $\mathscr{B}$.

(12) Let $K$ be a compact totally disconnected group. Let Aut $^{*}(K)$ be the automorphism group of $K$ which preserves small open normal subgroups of $K$. 
When does $\operatorname{Aut}^{*}(K)$ have a compactification, i.e., when is it maximal almost periodic?

(13) What is the structure of totally disconnected locally compact unimodular groups (if the Haar measure is a two-sided invariant)?

(14) Study the linear representation of totally disconnected locally compact groups over local fields.

(15) Assume $G$ is a totally disconnected locally compact group with maximal compact-open subgroups. Are there conjugacy properties among these subgroups (conjugacy under inner automorphisms or automorphisms)? (Note: If $G$ has a discrete uniform subgroup $\Gamma$, or $G / P$ carries a finite measure, then $G$ has maximal compact open subgroups.)

(16) What structure theorems can be deduced from results in analysis?

(17) Which conditions on abstract groups provide additional structures on locally compact groups?

\section{REFERENCES}

1. R. W. Bagley and T. S. Wu, Maximal compact normal subgroups and pro-Lie groups, Proc. Amer. Math. Soc. 9 (1985), 373-376.

2. R. W. Bagley, T. S. Wu, and J. S. Yang, On a class of topologically group more general than SIN groups, Pacific J. Math. 117 (1985), 209-217.

3. __ Pro-Lie groups, Trans. Amer. Math. Soc. 287 (1985), 829-838.

4. L_ Locally compact groups: Maximal compact subgroups and N-groups, Math. Proc. Cambridge Philos. Soc. 104 (1988), 47-64.

5. __ Compactly generated subgroups and open subgroups of locally compact groups, Proc. Amer. Math. Soc. 103 (1988), 969-976.

6. S. Grosser, O. Loos, and M. Moskowitz, Uber Automorphismengruppen lokalkompackter gruppen und derivationen von Lie-gruppen, Math. Z. 114 (1970), 321-339.

7. S. Grosser and M. Moskowitz, On central topological groups, Trans. Amer. Math. Soc. 1 (1967), 317-340.

8. $\frac{}{1-40}$, Compactness conditions in topological groups, J. Reine Angew Math. 246 (1971),

9. K. H. Hofmann, J. R. Liukkonen, and M. W. Mislove, Compact extensions of compactly generated nilpotent groups and pro-Lie, Proc. Amer. Math. Soc. 84 (1982), 443-448.

10. M. R. Pegrovian, Maximal compact normal subgroups, Proc. Amer. Math. Soc. 99 (1987), 389-394.

11. J. Tits, Automorphisms a deplacement borne des groupes de Lie, Topology 3 (1964), 97-107.

12. S. P. Wang, Compactness properties of topological groups, Trans. Amer. Math. Soc. 154 (1971), 301-314.

13. __ Compactness properties of topological groups. II, Duke Math. J. 39 (1972), 243-251.

14. $\ldots$, Compactness properties of topological groups. III, Trans. Amer. Math. Soc. 209 (1975), 399-418.

15. T. S. Wu, A certain type of locally compact totally disconnected topological groups, Proc. Amer. Math. Soc. 23 (1969), 613-614.

16. T. S. Wu and Y. K. Yu, Compactness properties of topological groups, Michigan Math. J. 19 (1972), 299-313.

17. __ Compactness conditions in topological groups, Michigan Math. J. 22 (1975), 269-280. 
18. R. W. Bagley, T. S. Wu, and J. S. Yang, Compact subgroups and compactly generated subgroups of topological groups, Proc. Amer. Math. Soc. (to appear).

19. B. A. F. Wehrfritz, Infinite linear groups, Ergebnisse der Math., Band 76, Springer-Verlag, 1973.

20. A. Borel and J. Tits, Groupes reductifs, Inst. Hautes Études Sci. Publ. Math. 27 (1965).

21. Karl H. Hofmann, Ta-Sun Wu, and Jeoung S. Yang, Equidimensional immersions of locally compact groups, Math. Proc. Cambridge Philos. Soc. (to appear).

Department of Mathematics, Case Western Reserve University, Cleveland, Ohio 44106 